\title{
¿Es costo-efectivo el rastreo de la diabetes tipo 2?
}

The cost-effectiveness of screening for type 2 diabetes. CDC Diabetes Cost-Effectiveness Study Group.

JAMA 1998;280:1757-63

\section{Objetivo}

La diabetes tipo 2 (DBT2) es una enfermedad seria y frecuente, y un tercio de los que la padecen no lo saben. Este estudio evalúa la costoefectividad de la detección y el tratamiento precoz en este grupo de pacientes.

\section{Diseño}

Se desarrolló un modelo de simulación de Monte Carlo* para calcular los costos y beneficios estimados de un único rastreo oportunista (realizado durante una visita de rutina al sistema de salud) para la DBT2 y compararlo con la práctica clínica actual. Se estimó la costo-efectividad para las personas de 25 años o más y para distintos grupos etarios. Los datos utilizados para el análisis provinieron de ensayos clínicos aleatorizados, estudios epidemiológicos y encuestas poblacionales.

\section{Lugar}

Perspectiva de un sistema de salud, EE.UU.

\section{Pacientes}

Cohorte hipotética de 10000 pacientes con DBT2 de reciente diagnóstico de la población general de EE.UU.

\section{Medición de resultados principales}

Costo por año de vida ganado y costo por QALY (año de vida ajustado por calidad)*

\section{Resultados}

El costo incremental para un sistema de salud del rastreo oportunista a todas las personas $\geq 25$ años de una cohorte hipotética de 10000 pacientes se estimó en $\$ 236449$ por año de vida ganado y $\$ 56649$ por QALY ganado. El rastreo fue más costo-efectivo en los jóvenes y disminuye a medida que aumenta la edad (entre los 25-34 años: \$35 768 por año ganado, 13 376/QALY, en los > 65 años: $\$ 575$ 241/QALY). Los beneficios de la detección temprana resultan principalmente del retardo en la aparición de complicaciones y la mejoría resultante de la calidad de vida, y no del aumento de la sobrevida. El modelo fue sensible a la prevalencia de DBT no diagnosticada, a la duración del intervalo prediagnóstico, y a la intensidad del régimen terapéutico (menor costo-efectividad del tratamiento intensivo).

\section{Conclusiones}

El diagnóstico y tratamiento temprano de la DBT2 a través del rastreo oportunista puede reducir la incidencia a lo largo de la vida de complicaciones microvasculares mayores y resultar en beneficios en cuanto a la calidad y cantidad de vida. El exceso de costos incurrido en el rastreo y tratamiento precoz está dentro de los rangos de otras intervenciones aceptadas, especialmente para el grupo de adultos jóvenes (población de alto riesgo de desarrollar complicaciones). Estos resultados sugieren que el rastreo es más costo-efectivo en los jóvenes.

\section{COMENTARIO}

Para justificar el rastreo poblacional de determinada enfermedad, dos jóvenes residentes plantearon los ya clásicos "criterios", ahora llamados de Frame y Carlson. ${ }^{1}$ Ellos postulan que la entidad debe ser una causa común de morbimortalidad (el caso específico de la diabetes lo cumple); debe ser tratable y detectable en su etapa asintomática (también lo satisface la DBT2: un tercio de los diabéticos no se saben enfermos, y habitualmente el periodo asintomático antes del diagnóstico es de 9 a 12 años, el cual podría ser reducido por el rastreo); deben haber tests efectivos para diagnosticarla (glucemia o HbA1c en este caso), el tratamiento temprano debe ser mejor que el tratamiento en la etapa de diagnóstico habitual (aun no está demostrado que le detección y tratamiento precoz de la DBT disminuya la morbimortalidad); y que el daño potencial de la intervención sea menor al del tratamiento precoz (las complicaciones de la DBT son irreversibles mientras que el tratamiento de la misma es efectivo y bastante seguro).

En el caso particular de la diabetes, las recomendaciones actuales de las fuerzas de tareas preventivas concluyen que no hay evidencia suficiente, ni a favor ni en contra, para la realización del rastreo a adultos asintomáticos (recomendación tipo "C").

La sistemática del rastreo evaluada en este estudio consiste en una glucemia en ayunas $\mathrm{y}$, de ser esta $>110 \mathrm{mg} / \mathrm{dl}$, un test de tolerancia oral a la glucosa para confirmar el diagnóstico. Más allá de la controversia del rastreo, el método más útil para realizarlo actualmente incluiría el dosa-

\section{Dr. Federico Augustovski}

Unidad de Medicina Familiar y Preventiva. Hospital Italiano de Buenos Aires.

\section{Referencias}

1. Frame PS, Carlson SJ. A critical review of periodic health screening using specific screening criteria. Part 1: Selected diseases of respiratory, cardiovascular, and central nervous systems. J Fam Pract 1975 Feb;2(1):29-36

2. Turner R, Holman R, Cull C et al. Intensive blood glucose control with sulphonilureas or insulin compared with conventional treatment and risk of complications in patients with type 2 diabetes (UKPDS 33). The Lancet 1998:352:837-53

3. The DCCT Research Group. The effect of intensive treatment of diabetes on the development and progression of long term complications in insulin dependent diabetes mellitus. N Engl.] Med 1993:329:977-86

4. Brown ML, Fintor L. Cost-effectiveness of breast cancer screening. Breast Cancer Res Treat 1993;25:113-18

5. Coppleson LW, Brown B. The prevention of carcinoma of the cervix. Am J 0bstet Gynecol 1976; 125:153-9

6. Littenberg B, Garber AM, Sox HC. Screening for hypertension. Ann Intern. 